\title{
LA INDUSTRIA DEL JUGUETE EN LA PROVINCIA DE ALICANTE
}

\author{
M. J. Santos Deltell
}

\section{RESUMEN}

El número de empresas dedicadas al sector juguetero en la provincia de Alicante es de 254. El mayor porcentaje de ellas corresponde a la Hoya de Castalla con $176(68,4 \%)$.

La población del sector asciende a 6.098 personas.

Onil e Ibi, son los dos municipios más representativos, cuentan con 138 factorías entre los dos y una plantilla de 4.821 trabajadores.

Predomina el tipo de planta empresarial de reducidas dimensiones, de hasta 25 empleados, con un porcentaje del 78,8\%. Sin embargo, ello no quiere decir que algunas sobrepasan este número, tal es el caso de Famosa en Onil, con 703, que está considerada como una de las más grandes de Europa.

\begin{abstract}
The number of factories dedicated to the toy sector in the province of Alicante in 1981 was 254. Among these, the greatest percentage corresponded to that of «La Hoya de Castalla» with 176 factories $(68,4 \%)$. Working population was simulated at 6.098 for the whale province. In Onil and Ibi therewere 138 factories withe 4.821 workers, both Onil and Ibi one the wast important villages wittin the sector.

The size of the average toy factory has, is reduced. A $78,8 \%$ of these have got up to 25 workers. However, there are exceptions, such as «Famosa» whose size is bigger. It is situated in Onil and it relies upon 703 workers and it is one of the leading toy factories in Europe.

This kind of industry has the effects of the entry of Spain into the E.E.C. with a reduction of exports and an increase of imports. The most affected industries will be the auxiliary ones, anyway no type of crisis can be anticipated in high quality toys given its high competivity.
\end{abstract}


El juguete en Alicante se encuentra localizado en un área muy concentrada, cuya zona principal es la zona de Castalla. Esta comarca es el núcleo más representativo, y dentro de ella, existen dos modalidades; Ibi se ha especializado en juguetes de todas clases, cuyas principales materias primas son el plástico y la chapa metálica, y en Onil la producción se ha centrado en torno a la muñeca de calidad media-alta, cuya principal materia prima es el plástico.

Esta comarca integrada por los dos municipios citados y por Castalla y Tibi, agrupaba en 1981 un total de 176 empresas jugueteras repartidas del siguiente modo: 50,3\% en Ibi (88), 28,2\% en Onil (50), 19,8\% en Castalla (35) y 1,7\% en Tibi (3).

Mientras que Castalla ha seguido el impulso fabril de su vecino Onil, y básicamente lo que produce es muñecas, Tibi se ha orientado hacia el juguete, fabricando sobre todo piezas sueltas, y accesorios. Cuenta con talleres de maticería que trabajan para el núcleo cercano de Ibi. Este tipo de industrias auxiliares funcionan al servicio de pequeñas empresas que no disponen del capital capaz de afrontar los diversos gastos de financiación que se requiere para dar por terminado el proceso de fabricación de un juguete. La época de máxima expansión de este tipo de industrias auxiliares coincide con la década de los años 60, es decir, con la época dorada de esta industria, destacando los talleres de inyección de plástico, fábricas de engranaje para mecanismos y resortes mecánicos e industrias de cartón en Tibi y Castalla.

El juguete de tipo educativo construido básicamente con madera, predomina en Denia con un total de 36 empresas y es este núcleo después de la «Foia» el más importante centro del sector juguetero alicantino. En el resto de la provincia las empresas dedicadas a este ramo, están más diversificadas. Las demás localidades provinciales donde aparecen este tipo de industrias son: 


\begin{tabular}{|c|c|c|c|c|}
\hline Año 1981 & $N^{\circ}$ Empresas & & & apresas \\
\hline Alcoy & 3 & "Foia" & & 176 \\
\hline Alfaz del Pi & 1 & Denia & & 54 \\
\hline Alicante & 4 & TOTALES & & 230 \\
\hline Almoradi & 1 & & & \\
\hline Bañeres & 4 & & & \\
\hline Benejama & 1 & & & \\
\hline Beniarbeig & 1 & & $\mathrm{~N}^{\circ}$ Empresas & $\%$ \\
\hline Benijófar & 1 & "Foia" & 176 & 68,4 \\
\hline Biar & 3 & Denia & 50 & 20,8 \\
\hline $\begin{array}{l}\text { Calpe } \\
\text { Campo de Mirra }\end{array}$ & $\begin{array}{l}1 \\
1\end{array}$ & Resto & 28 & 10.8 \\
\hline Elche & 1 & TOTALES & 254 & 100.0 \\
\hline Novelda & 1 & & & \\
\hline Ondara & 3 & & & \\
\hline Penáguila & 1 & & & \\
\hline Torremanzanas & 1 & & & \\
\hline TOTALES & 28 & & & \\
\hline
\end{tabular}

En la mayoría de los municipios donde aparece alguna representación del mundo del juguete, más que de empresas se trata de talleres que tienen un promedio de 1 a 5 empleados; tan sólo en algunos pueblos, como es el caso de la «Foia», estas reducidas dimensiones adquieren mayores proporciones, llegando a un promedio de 50 empleados/empresa.

Concentración espacial del sector

Una de las características de la industria valenciana es su concentración en lugares muy concretos, geográficamente reducidos con un número alto de empresas dedicadas a la producción de una misma rama industrial, como es el caso del juguete.

Ello crea condiciones especiales de cara a posibles resoluciones de sus problemas. Este modo de distribución permite acometer acciones conjuntas en mejores condiciones que cuando los sectores se hallan distribuidos en amplias zonas geográficas en las que resulta problemático poner en contacto a responsables de empresas, y conjugar intereses que frecuentemente no resultan coincidentes. 


\section{Localización}

Todos los municipios que cuentan con empresas dedicadas a este sector se encuentran en la mitad norte provincial a excepción de Elche, Benijófar y Almoradí, situados en las comarcas meridionales del Bajo Vinalopó y del Bajo Segura respectivamente. Hay que advertir que más que fábricas, lo que existe en estas localidades son talleres, pues sólo hay 3 trabajadores empleados en cada uno.

El área del juguete se localiza principalmente en la «Foia» de Castalla, que comprende los municipios de Castalla, Ibi, Onil y Tibi y en la vecina ciudad de Biar que pertenece a la comarca del Alto Vinalopó, pero que dista tan sólo unos kilómetros de la «Foia». A estos cinco municipios vamos a denominarlos zona A; agrupa 179 empresas y 5.454 empleados $(91,2 \%)$. La zona B estaría formada por los pueblos de: Alcoy, Bañeres, Benejama, Campo de Mirra y Penáguila, con un total de 10 empresas y 89 empleados. Todos estos núcleos constituyen el foco principal de la juguetería y los hemos agrupado debido a su proximidad. De todas formas salta a la vista la importancia mayor de la zona A con respecto a la zona B.

Otro centro juguetero a destacar sería el formado por Denia y poblaciones cercanas: Calpe, Ondara y Beniarbeig, donde existen 59 empresas y 440 empleados.

Por último existen centros aislados unos de otros que también cuentan con alguna representación de la industria del juguete, y la capital provincial: Alicante. Estas poblaciones son Elche, Alfaz del Pi, Almoradí, Benijófar, Novelda y Torremanzanas, con 10 empresas jugueteras y 115 empleados.

\section{Localización industrial (año 1981)}

\section{1) Foco Principal}

№ Empresas $\quad$ № Empleados

A) Castalla $\quad 35$

Ibi 88

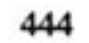

Onil 50

Tibi

Biar

B) Alcoy

Banteres 
2) Núcleo de Denia

Denia

Calpe

Ondara

Beniarbeig

\section{3) Núcleos aislados}

Alfaz del Pi

$$
1
$$

Alicante

4 95

Almoradi

\section{1}

Benijófar

Elche

Novelda

Torremanzanas

Totales

\section{Población Laboral}

La población más numerosa de la juguetería se encuentra en la Hoya de Castalla. En Ibi asciende a un $51 \%$ y en Onil casi a un $30 \%$ con respecto al total de población activa. Ello significa que un $80 \%$ de los trabajadores de esta industria se localizan en estos dos pueblos, lo que da una prueba de su importancia dentro del sector.

\begin{tabular}{|c|c|c|}
\hline - & Población Laboral & $\%$ Total \\
\hline Ibi & 2.939 empleados & $50,4 \%$ \\
\hline Onil & 1.882 empleados & $29.5 \%$ \\
\hline TOTAL & 4.821 empleados & $79,9 \%$ \\
\hline
\end{tabular}

El resto se reparte entre los demás municipios correspondiendo los porcentajes más altos a Castalla: $7 \%$, Denia 6,5\%, Biar 2,58\% y 1,4\% en Alicante.

De la misma forma que Ibi es el municipio juguetero por antonomasia en el marco provincial y va a la cabeza en el número de empresas y en el de empleados, también lo es en cuanto a inversión de bienes de equipo con 1.077.000 millones de pts. Lo que representa un $66,3 \%$ con respecto al total de lo invertido en el juguete a nivel provincial.

A Ibi le sigue Onil, donde la inversión es tres veces menor, ascendiendo ésta a 337.000 millones de pts. y representando un 20,7\%. Estos dos pueblos son los que más dinero invierten en este tipo de industrias, aunque existe una diferencia 
y es que mientras planta muñequera resulta más económica, la de juguetes resulta más costosa, siendo por ello el porcentaje de inversión en Onil menor con respecto a Ibi. El encarecimiento del costo de la inversión de las empresas jugueteras se relaciona con el tipo de materia prima empleada, siendo más costosas las piezas de metales que las de plástico.

Con porcentajes inferiores de inversión figuran Castalla $(4,30 \%)$, Denia $(3,56 \%)$ y el resto de los municipios comprendidos entre los valores de Biar $(1,70 \%)$ y Elche $(0,01 \%)$.

№. Empresas, población laboral y nivel inversión.

\begin{tabular}{|c|c|c|c|c|c|c|}
\hline Municipios & Empresas & $\%$ & Empleados & $\%$ & Inversión & $\%$ \\
\hline Ibi & 88 & 34.3 & 2.939 & 50.4 & 1.077 & 66.3 \\
\hline Onil & 50 & 19,3 & 1.882 & 29.5 & 377 & 20,7 \\
\hline Castalla & 35 & 13.5 & 444 & 6.96 & 70 & 4,3 \\
\hline Tibi & 3 & 1.1 & 24 & 0.3 & 2 & 0.1 \\
\hline Denia & 54 & 20.8 & 418 & 6.5 & 58 & 3,5 \\
\hline Alcoy & 3 & 1.1 & 39 & 0.6 & 9 & 0.5 \\
\hline Alfaz del $\mathrm{Pi}$ & 1 & 0.3 & 5 & 0.07 & 0,2 & \\
\hline Alicante & 4 & 1.5 & 95 & 1.4 & 26 & 1,6 \\
\hline Almoradi & 1 & 0.3 & 3 & 0.04 & 0.2 & \\
\hline Bañeres & 4 & 1.5 & 23 & 0.36 & 3 & 0.2 \\
\hline Biar & 3 & 1.1 & 165 & 2.58 & 27 & 1.7 \\
\hline Campo de Mirra & 1 & 0.3 & 2 & 0,03 & 0.2 & \\
\hline Ondara & 3 & 1.1 & 5 & 0.07 & 2 & 0,1 \\
\hline Elche & 1 & 0.3 & 3 & 0.04 & 0.2 & 0,01 \\
\hline Benejama & 1 & 0.3 & 21 & 0.32 & 0.7 & 0,05 \\
\hline Beniarbeig & 1 & 0.3 & 11 & 0.17 & 5 & 0,34 \\
\hline Benijófar & 1 & 0.3 & 1 & 0.01 & 0.2 & \\
\hline Calpe & 1 & 0.3 & 6 & 0.09 & 1 & 0,10 \\
\hline Novelda & 1 & 0.3 & 3 & 0.04 & 0.5 & 0.03 \\
\hline Penáguila & 1 & 0.3 & 4 & 0.06 & 3 & 0.18 \\
\hline Torremanzanas & 1 & 0.3 & 5 & 0.07 & 1 & 0,07 \\
\hline TOTALES & 258 & & 6.098 & & $\begin{array}{c}1.623,2 \\
\text { millones }\end{array}$ & \\
\hline
\end{tabular}

Fuente: Delegación de Industria. Alicante. 
Hay que hacer notar el hecho de que tanto en Alicante como en Biar, la inversión realizada en este tipo de industria (26 y 27 millones de pts. respectivamente) es bastante elevada, si observamos el número de empresas, lo que facilita un promedio de 6 millones por empresa en el caso de Alicante y de 9 en el de Biar. En Castalla se han invertido 70 millones de pts. para 35 empresas, lo que supone una inversión de 2 millones/fábrica; en Denia aún es menor, 50 millones han servido para 54 empresas, resultando una relación de 1,1 millones por empresa. En Onil e Ibi la relación se establecería del siguiente modo: 6,72 millones en Onil y 12,10 en Ibi.

\section{Dimensiones de las empresas}

En el tamaño de la planta juguetera predomina la de reducidas dimensiones, es decir, de hasta 25 empleados con un porcentaje del $78,8 \%$ con respecto al total, tamaño similar a la de los países del C.E.E.

La industria del juguete manifiesta un claro predominio de la pequeña y mediana empresa. Es un tipo de industria que muestra gran atomización en cuanto al número de fabricantes con un reducido tamaño empresarial, pero con una gran concentración geográfica de ubicación de empresas.

Este tipo de empresas representa un 28,3\% en Ibi, 19,4\% en Onil, 14,4\% en Castalla, $25,8 \%$ en Denia, $0,9 \%$ en Alcoy y Biar, $1,4 \%$ en Alicante y un $100 \%$ en Tibi, Alfaz del Pi, Almoradí, Benijófar, Bañeres, Novelda, Ondara, Elche, Benejama, Beniarbeig, Calpe, Campo de Mirra, Penáguila y Torremanzanas.

El grupo siguiente de empresas más numerosas sería el que cuenta entre 25 y 50 empleados con un $9,8 \%$ de representación a nivel provincial. Se encuentra repartido por los principales núcleos jugueteras: Ibi 12 empresas, Onil y Castalla con 5 cada una, Denia 2 y Alcoy 1.

A continuación y con porcentajes muy próximos se encuentran los grupos de 50 a 100 empleados con un 5,4\% y de 100 a 250 con un 4,7\%. En el primero destaca el municipio de Ibi con 10 empresas, seguido de Onil con 2 y Castalla y Alicante con una cada uno. En el de 100 a 250 empleados sigue estando Ibi en primer lugar con ocho fábricas de estas dimensiones, en Onil hay tres y una en Biar.

Por último, con más de 250 trabajadores hay 2 empresas, una en Ibi y otra en Onil. Rico, S. A. en Ibi, situada en la calle de Colón, $n^{\circ}$. 80, cuenta con 360 empleados y Famosa en Onil, carrer de San Antoni, 9, supera con creces los 250, ya que en la actualidad tiene 713 obreros.

Fuera de la Hoya de Castalla, destacan como empresas jugueteras más grandes una en Biar con 153 personas empleadas, Muñecas Jesmar creada en 1980; otra en Alicante con 127 trabajadores: Sociedad Valenciana del Juguete S.A. Valtoy, del año 1978. En Denia hay una que cuenta con un personal integrado por 43 trabajadores, Sociedad Valenciana del Juguete S.A. Valtoy.

Los talleres de pequeñas dimensiones se localizan en el resto de los municipios que aparecen en el cuadro VII. 
Dimensiones de las empresas.

\begin{tabular}{lrrc} 
& N. & \multicolumn{1}{c}{$\%$} & $N^{\circ}$. Empleados \\
Menores de 25 & 205 & 78.8 & 1.463 \\
$25-50$ & 25 & 9.8 & 901 \\
$50-100$ & 14 & 5.4 & 861 \\
$100-250$ & 12 & 4.7 & 1.800 \\
Más de 250 & 2 & 1.1 & 1.073 \\
TOTALES & 258 & 100 & 6.098
\end{tabular}

Empresas menores de 25 empleados Empresas de 25 a 50 empleados

\begin{tabular}{|c|c|c|c|c|c|}
\hline Municipios & Fähricas & Personal & Municipios & Fäbricas & Personal \\
\hline Ibi & 57 & 441 & Ibi & 12 & 431 \\
\hline Onil & 39 & 340 & Onil & 5 & 174 \\
\hline Castalla & 29 & 187 & Castalla & 5 & 185 \\
\hline Tibi & 3 & 24 & Denia & 2 & 78 \\
\hline Denia & 52 & 340 & Alcoy & 1 & 33 \\
\hline Alcoy & 2 & 6 & TOTALES & 25 & 901 \\
\hline Alfaz del $\mathrm{Pi}$ & 1 & 5 & & & \\
\hline Alicante & 3 & 21 & & & \\
\hline Almoradi & 1 & 3 & & & \\
\hline Bañeres & 4 & 23 & & & \\
\hline Biar & 2 & 12 & & & \\
\hline Campo de Mirra & 1 & 2 & Empresas de & 50 a 100 & empleados \\
\hline Ondara & 3 & 5 & & & \\
\hline Elche & 1 & 3 & Municipios & Fábricas & Personal \\
\hline Benejama & 1 & 21 & Ibi & 10 & 559 \\
\hline Beniarbeig & 1 & 11 & Onil & 2 & 156 \\
\hline Benijöfar & 1 & 1 & Castalla & 1 & 72 \\
\hline Calpe & 1 & 6 & Alicante & 1 & 74 \\
\hline Novelda & 1 & 3 & TOTALES & 14 & 861 \\
\hline Penáguila & 1 & 4 & & & \\
\hline Torremanzanas & 1 & 5 & & & \\
\hline TOTALES & 205 & 1.463 & & & \\
\hline
\end{tabular}




\begin{tabular}{lcrlcr} 
Empresas de & 100 a 250 & empleados & \multicolumn{3}{l}{ Empresas de más de 250 empleados } \\
Municipios & Fábricas & Personal & Municipios & Fábricas & Personal \\
Ibi & 8 & 1.148 & Ibi & 2 & 360 \\
Onil & 3 & 499 & Onil & 1 & 713 \\
Biar & 1 & 159 & TOTALES & 2 & 1.073 \\
TOTALES & 12 & 1.806 & & &
\end{tabular}

Entre las empresas menores de 25 empleados existen un total de 205 que cuentan con una población laboral de 1.463 empleados repartidos prácticamente por todo el territorio provincial, 17 municipios, de estos, 7 cuentan con una empresa de estas características, dos con dos fábricas, tres pueblos con tres, y uno con cuatro (Bañeres). Los cuatro municipios restantes (Ibi, Onil, Castalla y Denia), se reparten las 177 que faltan.

Municipios que cuentan con empresas que tienen entre 25 y 50 empleados hay 5 , con un total de 25 fábricas y 901 empleados. De estas, 12 corresponden a Ibi y el resto se reparte del modo siguiente: 5 en Onil y en Castalla, 2 en Denia y 1 en Alcoy.

Entre 50 y 100 empleados destacan Ibi con 10 empresas, Onil con dos y Castalla y Alicante con 1, lo que hace un total de 14 fábricas y 861 empleados.

De 100 a 250 trabajadores existen 12 empresas en toda la provincia con un total de 1.806 trabajadores. De éstas, 8 pertenecen a Ibi, 3 a Onil y 1 a Biar.

Por último, empresas superiores a los 250 empleados, sólo se encuentran en la «Foia», hay 1 en Ibi y una en Onil, con un total de 1.073 empleados.

\section{Situación actual}

Entre las características que pueden definir a la industria del juguete podemos citar las siguientes: manifiesta un claro predominio de la pequeña y mediana empresa $(78,8 \%)$; alta concentración geográfica y de la producción en la provincia de Alicante; en 1981 la producción de juguetes de toda España fue de 28.000 millones de pesetas, de los que la mitad correspondió a la provincia de Alicante (54,63\%).

La mayor concentración de mano de obra es también alicantina, ya que ocupa a más de la mitad del personal fijo que trabaja en esta industria. Produce más de la mitad de la facturación nacional y sin embargo cuenta en su zona con un poco más de la cuarta parte de empresas jugueteras nacionales (1).

La principal característica del empleo generado por esta industria es que tienden a ser de temporada (el 54\% de los empleados en la campaña Navidad-Reyes son eventuales, prefiriendo el personal femenino al masculino entre los temporeros) (2). Se trata de un sector preparado para producir más cuando el mercado lo requiera, por el carácter cíclico de las ventas, la industria del juguete exige acumulación de stocks en las fechas punta con el consiguiente riesgo de 
excedentes de producción y de financiación. El sector juguetero cuenta con unas características peculiares y específicas, por un lado la sombra perecedera que lleva el juguete tras de sí, salvo casos muy excepcionales los juguetes pasan rápidamente de moda (excepción: la muñeca Nancy de Famosa, con más de veinte años de vigencia en el mercado). Por ello el fabricante debe calcular meticulosamente la longitud de la serie que va a lanzar y la inversión que conllevará el lanzamiento de este juguete en maquinaria y utillaje, todo ello encaminado a amortizar esos gastos antes de que el artículo quede obsoleto.

Salvo en el caso de las muñecas, donde la especialización es mayor ya que la diferenciación del producto se obtiene a través del diseño y por la publicidad de la marca, en los demás casos la producción es bastante artesanal con altos niveles de empleo y baja productividad. También se está concediendo cada vez más importancia al tipo de envase, lo que se patentiza a la hora de establecer relación calidad-precio. De la misma forma que se está asistiendo hoy en día a una mayor necesidad de cualificación en la mano de obra que va constantemente en aumento.

\section{Mercado exterior}

En cuanto a sus relaciones con el exterior, ha sido una industria muy protegida arancelariamente con cupos limitados de importaciones por lo que no ha tenido que enfrentarse con la competencia extranjera dentro del mercado español. Hasta ahora los aranceles restringían al máximo las importaciones del juguete pero con la adopción del tratado económico comunitario (T.E.C.) y con la entrada de nuestro país en la Comunidad Económica Europea (C.E.E.) el nivel de protección del juguete español es bajo con relación al resto de los países comunitarios. Sin embargo, España participa con un $20 \%$ de sus exportaciones dentro del Mercado Común. La exportación de juguetes y muñecas de la «Foia» durante 1985 fue de 6.321 millones.

El ingreso de España en el mercado comunitario va a suponer que aumenten las importaciones de juguetes de alta calidad, hecho que se viene observando desde la firma del acuerdo de España y la C.E.E. en 1970 y además se espera un recrudecimiento de la situación difícil para las empresas marginales o de tipo pequeño que son las más abundantes. Existe una incidencia distinta sobre el tipo de juguete, los más perjudicados son los de baja calidad o baratijas, porque cuentan con la competencia de los del SE asiático. Se observa en la actualidad un aumento lento y sistemático de la participación de terceros países en el mercado español (artículos baratos, electrónicos y de artesanía procedentes del SE asiático).

La oferta española actual tiende a una mayor concentración dentro del sector del juguete de plástico y mecánico de tipo medio. En el campo de la muñeca de calidad es probable que no se produzcan daños dado que hasta el presente, las muñecas españolas son altamente competitivas. La integración en el Mercado Común Europeo puede suponer una beneficiosa ampliación del mercado si se 
adopta una política de comercialización adecuada. El daño puede producirse en la muñeca barata y en las empresas pequeñas que fabrican este tipo de artículos de bajo precio, puesto que no se puede soportar la competencia con los nuevos países industriales (N.P.I.), (Taiwan, Corea, Hong-Kong, Singapur, Brasil y México) que disponen de mano de obra abundante y más barata que en España (80\%), tecnología adecuada y en algunos casos materias primas y energéticas. 


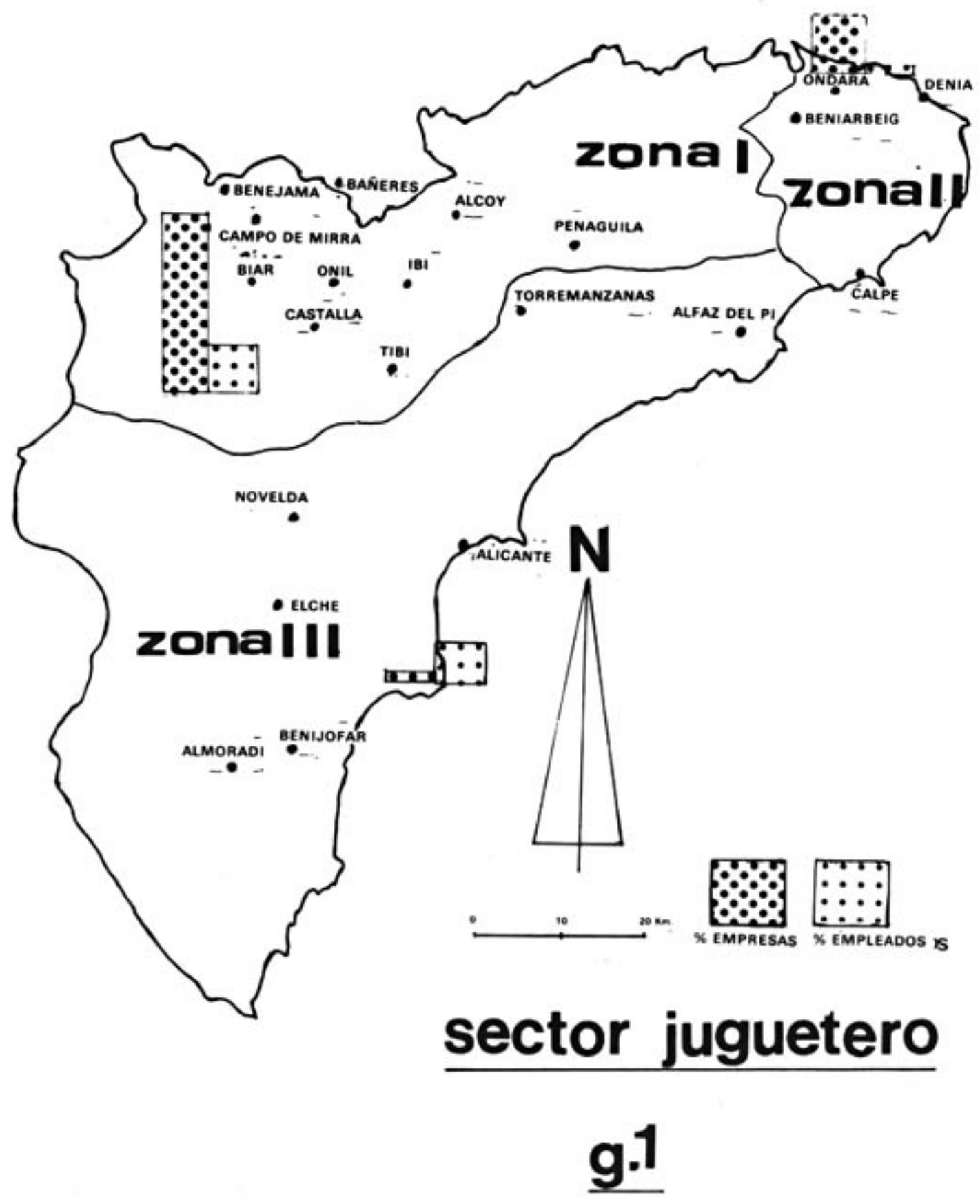



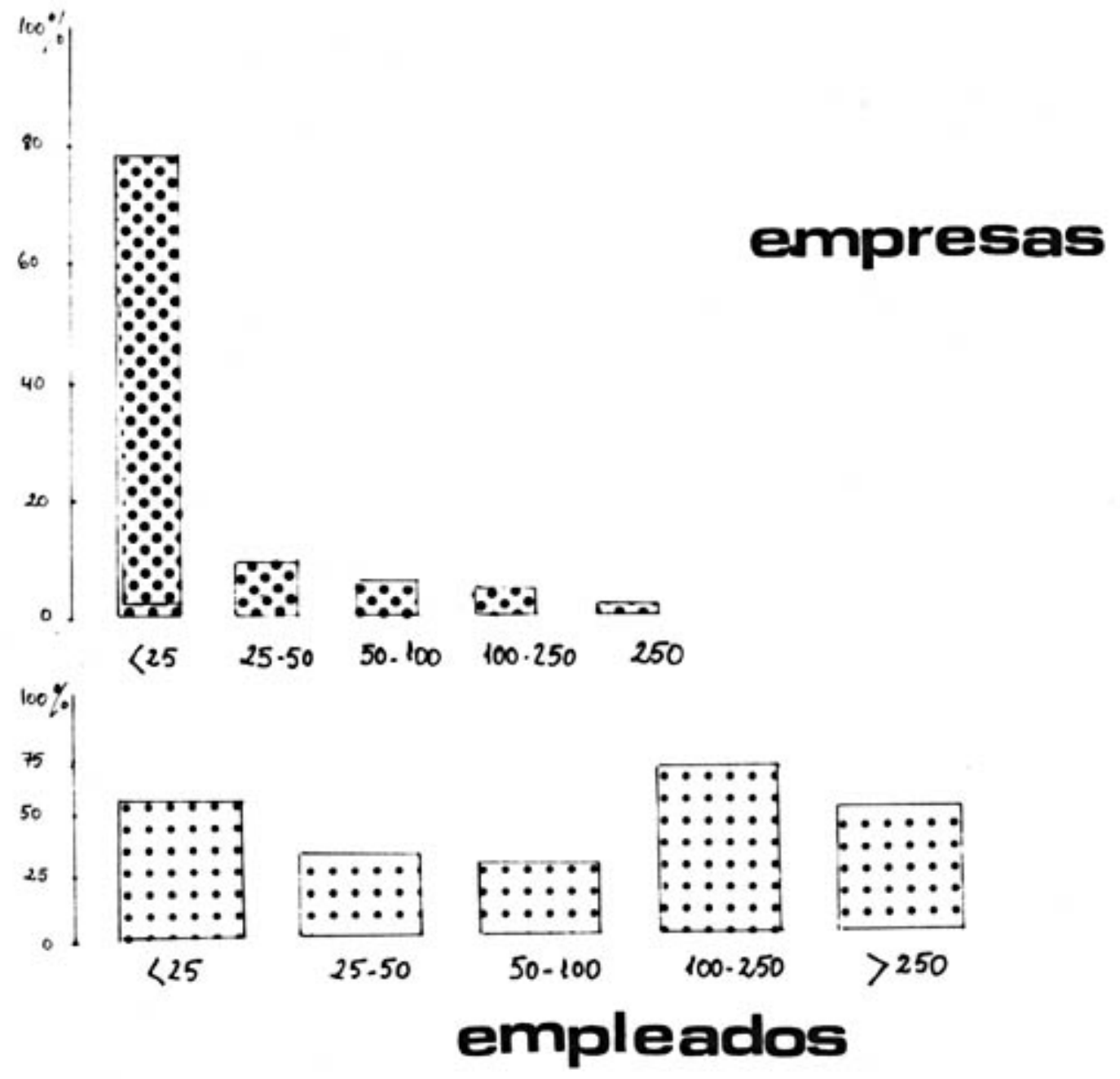

g. 2 


\section{NOTAS}

1. Según la Asociación Española de Fabricantes de Juguetes, en 1979 había 348 empresas en toda España, 94 pertenecen a la «Foia» $(27,01 \%)$.

2. En 1978 habría en Onil 2.000 empleados, de los que 1.093 eran temporeros. 


\section{PUBLICACIONES DEL INSTITUTO UNIVERSITARIO DE GEOGRAFÍA}

VARIOS. I Coloquio sobre la Propiedad Agraria en España. 481 pág. Alicante, 1981.

BELANDO CARBONELL, R. Estudio demográfico de Monóvar Siglos XVI-XX. 191 pág. Alicante, 1983.

GIL OLCINA, A. y otros. Lluvias torrenciales e inundaciones en Alicante. 128 pág. 30 lám. Alicante, 1983.

GOZÁLVEZ PÉREZ, V. Crevillente. Estudio urbano, demográfico e industrial, (en colaboración con el Ayuntamiento de Crevillente), 1983, 189 pág.

GUMUZZIO, J. y MATARREDONA, E. Formaciones edáficas del Campo de Alicante, 51 pág. 5 lám., Alicante, 1983.

PONCE HERRERO, G. Sax, población y potencial económico, 194 pág. Alicante, 1986.

GIL OLCINA, A. y otros. Inundaciones en la ciudad y término de Alicante. 120 pág. Alicante, 1987.

JUÁREZ SÁNCHEZ-RUBIO, C.; PONCE HERRERO, G., y VERA REBOLLO, J. F. La Universidad de Alicante y su área geográfica de atracción. Secretariado de Publicaciones de la Universidad de Alicante, 1987.

Revista: «Investigaciones Geográficas». Anales de la Universidad de Alicante. Números 00301(1983), 2(1984), 3(1985), 4(1986), 5(1987).

Distribución de ejemplares: Secretariado de Publicaciones de la Universidad (Alicante).

\section{PUBLICACIONES EN COLABORACIÓN CON EL INSTITUTO «GIL ALBERT» DE LA EXCMA. DIPUTACIÓN PROVINCIAL DE ALICANTE}

CRESPO GINER, J. San Juan: estudio demográfico-económico de un municipio de la Huerta de Alicante, 113 pág. 16 lám. Alicante, 1979.

MATARREDONA COLL, E. El Alto Vinalopó. Estudio geográfico, 370 pág. 35 lám. Alicante, 1983.

RAMOS HIDALGO. A. Evolución urbana de Alicante, 323 pág. 45 lám. Alicante, 1984.

VERA REBOllo, F. Tradición y cambio en el Campo del Bajo Segura. 219 pág. 21 lám. Alicante, 1984.

GOZÁLVEZ PÉREZ, V. y otros, Atlas sociodemográfico de la ciudad de Alicante, 1987, 92 pág. 47 mapas, f.t.

MARCO MOLINA, J. A. El Macizo del Maigmó. Estudio de Geografía física. Instituto de Estudios «Juan Gil Albert», Alicante, 1987. 171 pág.

BOX AMORÓS, M. Humedades y áreas lacustres de la provincia de Alicante, Instituto de Estudios «Juan Gil Albert»-C.S.I.C., 1987, 290 pág.

VERA REBOLLO, J. F. Turismo y urbanización en el litoral alicantino, Instituto Universitario de Geografía-Instituto de Estudios «Juan Gil Albert»-Secretariado de Publicaciones de la Universidad de Alicante y Caja de Ahorros de Alicante y Murcia, Alicante, 1987, 441 pág.

Distribución de ejemplares del Instituto «Juan Gil Albert» de la Diputación.

$$
\begin{gathered}
\text { LA TIERRA LIBROS } \\
\text { C/. Pintor Gisbert } \\
\text { Alicante }
\end{gathered}
$$

